\title{
OPTIMIZATION OF THE COMPUTATION OF MULTIDIMENSIONAL INTEGRALS FOR ESTIMATING THE MESON LIFETIME
}

\author{
D. Goderidze ${ }^{\text {a }}$, Yu.L. Kalinovsky, A.V. Friesen \\ Joint Institute for Nuclear Research, 6 Joliot-Curie, Dubna, 141980, Russia \\ E-mail: ${ }^{a}$ goderidze@jinr.ru
}

To calculate the lifetime of mesons in hot and dense nuclear matter, it is necessary to compute multidimensional integrals with a complicated integrand function. This work presents an algorithm and methods to calculate such integrals on the basis of the Monte-Carlo method. To optimize the computation, the parallel calculation algorithm is implemented in the $\mathrm{C}++$ programming language using OpenMP and NVIDIA CUDA technologies. The calculations are performed on nodes with multicore CPUs and Intel Xeon Phi coprocessors and the Nvidia Tesla K40 accelerator installed within a heterogeneous cluster of the Meshcheryakov Laboratory of Information Technologies, Joint Institute for Nuclear Research, Dubna. The code is used to calculate the pion lifetime using all possible pionpion scattering modes.

Keywords: meson lifetime, pion damping, Monte Carlo, parallelization

Daviti Goderidze, Yuri Kalinovsky, Alexandra Friesen

Copyright (C) 2021 for this paper by its authors. Use permitted under Creative Commons License Attribution 4.0 International (CC BY 4.0). 


\section{Meson lifetime}

The investigation of particles and their properties, including scattering and decay modes, the lifetime and width of particles in critical conditions of heavy ion collisions, is an important problem in modern physics. The theoretical description of strong-interaction matter under critical conditions is a complicated task, and it is not always possible to find an analytical solution to the problem. Therefore, the use of modern computer technologies plays a key role in theoretical research.

This paper is devoted to the investigation of the pion damping width in a hot pion gas within the NJL model. The main contribution to the width/lifetime comes from pion-pion collisions. To calculate the meson lifetime in hot and dense nuclear matter, it is necessary to compute 5-dimensional integrals with a complicated integrand function. This work presents an algorithm and methods for the calculation of complicated integrals based on the Monte-Carlo method using the parallelization algorithm to optimize the computation time.

The meson lifetime $\tau$ is determined by the particle self-energy [1]:

$$
\Gamma=\tau^{-1}=\Sigma^{>}(p) \pm \Sigma^{<}(p),
$$

where the sign depends on the type of the particles: «+» for fermions and «-» for bosons. The $\Sigma^{>}$ function is responsible for direct processes, when a particular state decays into other states. The $\Sigma^{<}$ function is responsible for inverse processes that can restore the decayed state due to the decay of other particles to this state again. This process prolongs the lifetime of particles in the medium. The $\Sigma$ functions have a complicated form:

$$
\Sigma^{>(<)}(p)=\frac{1}{(2 \pi)^{3}} \int d \Omega \int \frac{d \vec{p}_{1}}{2 E_{1}} \frac{1}{8 \pi} \int_{-1}^{1} d(\cos \alpha) \frac{\left|\vec{p}_{3}\right|^{2}}{\left|\vec{p}_{3}\right|^{2}\left(\sqrt{s_{2}}+m_{1}\right)-\left|\vec{p}_{1}\right| E_{3} \cos \alpha}|A|^{2} F^{>(<)},
$$

where $d \Omega=d s_{1} A\left(s_{1}\right) d s_{3} A\left(s_{3}\right) d s_{4} A\left(s_{4}\right)$, the spectral function is calculated in the BreitWigner form $A\left(s_{i}\right)=2 M_{i} \Gamma_{i}\left(\left(s_{i}-M_{i}^{2}\right)^{2}+M_{i}^{2} \Gamma_{i}^{2}\right)^{-1}$ with $s_{i}=p_{i}^{2}$. The factors $F^{>}=$ $n_{1}\left(n_{3}+1\right)\left(n_{4}+1\right)$ and $\quad F^{<}=\left(n_{1}+1\right) n_{3} n_{4}$ are introduced for brevity, and $n_{i}$ are the particle distribution functions $n_{i}=\left(\exp \left(\beta \sqrt{s_{i}+\bar{p}^{2}}\right) \pm 1\right)^{-1}$ according to the particle statistics $[1,2]$. The momenta $\vec{p}_{3}$ are defined as:

$$
\left|\vec{p}_{3}\right|_{1,2}=\frac{\left|\vec{p}_{1}\right| a \cos \alpha \pm \sqrt{\left|\vec{p}_{1}\right|^{2} a^{2} \cos ^{2} \alpha+\left(\left(\sqrt{s_{2}}+E_{1}\right)^{2}-\left|\vec{p}_{1}\right|^{2} \cos ^{2} \alpha\right)\left(a^{2}-4 s_{3}\left(\sqrt{s_{2}}+E_{1}\right)^{2}\right)}}{2\left(\left(\sqrt{s_{2}}+E_{1}\right)^{2}\right)-\left|\vec{p}_{1}\right|^{2} \cos ^{2} \alpha}
$$

with $a=\left(\sqrt{s_{2}}+s_{1}\right)^{2}+s_{3}-s_{4}+s_{1}-E_{1}^{2}$.

To calculate the meson lifetime, the scattering amplitude $|A|$ of the process under investigation should be calculated. The amplitude $|A|$ is generally considered as an invariant amplitude and depends on the Mandelstam variables $s, t, u$, where $s+t+u=\sum s_{i}$.

It is seen from Eq. (2) that the structure of the integrand function is complicated and depends on many variables. The complex structure of the integrand is due to the kinematic conditions of physical processes and is limited by the pole in the denominator and the negative value of the radical expression. To show the 5-dimensional integration domain for the integral (2), we transfer it to the plane $\left(\vec{p}_{1}, \vec{p}_{3}\right)$, since by generating the arbitrary point $\left(s_{1}, s_{3}, s_{4}, \vec{p}_{1}, \cos \alpha\right)$ it is possible to determine $\vec{p}_{3}$ for the fixed $\vec{p}_{1}$ (Fig. 1).

\section{Optimization of computing}

In this work, the Monte Carlo (MC) method [3] is used to calculate the integrals in Eq. (2). It is well known that the accuracy of the MC method depends on the number of selected points, as well as on the quality of the random number generator. Tina's Random Number Generator (TRNG) [4] is chosen for calculations as it has various distributions (uniform and non-uniform) and is also optimized for using in parallel computations. Parallel calculations are required for the integrals in Eq. (2), and the result and accuracy strongly depend on the number of selected points and the best convergence is 
achieved after the number of points reaches $2 \cdot 10^{7}$ (Fig. 2, left). The optimization of computations is carried out in two directions: simplification of the functions and use of parallelization technology.

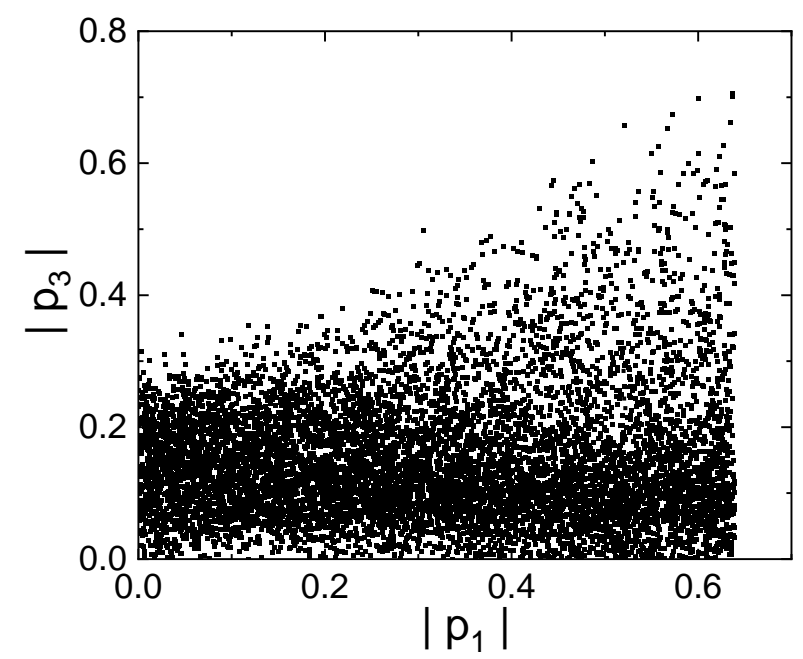

Figure 1 . Behavior of the function in the integration area using the $\left(\vec{p}_{1}, \vec{p}_{3}\right)$ variables and fixing $\cos \alpha=0$.

To obtain the selected point in the integration domain of Eq. (2), it is necessary to generate 5 random numbers. Taking into account the fact that $\cos (\alpha)$ changes in $[-1,1]$, the reflection of the selected $\cos (\alpha)$ from $[0,1]$ to $[-1,0]$ relative to zero, while saving the other variables, reduces the required number of generations twice. Since $\Sigma^{>}$and $\Sigma^{<}$differ by the factor $F^{>(<)}$, the positions of the special points for these functions are the same, which allows calculating the functions $\Sigma$ simultaneously to reduce the total computation time twice. This method reduces the number of the generated points and the total number of the required calculations by 4 times, thus accelerating the computation process.

According to the standard MC method, if the accuracy of the integral calculated over $\mathrm{N}$ selected points is insufficient, the number of points should be increased. To optimize calculations, the additional number of the required selected points is equal to the increase step $\mathrm{M}$. The calculated results for $\mathrm{M}$ points are added to the previous calculations to get the final answer, and the accuracy is checked for $\mathrm{N}+\mathrm{M}$ points.

Parallelization is also used to accelerate the machine time for computations. For this purpose, the number of generated points $(\mathrm{N})$ is evenly distributed among all threads $(\mathrm{P})$. The remainder of the points $(\mathrm{N} \bmod \mathrm{P})$ is also additionally distributed over the required number of threads (instead of sending it to one thread). The program is implemented for computations in both single-thread and multi-thread versions. The multi-thread version uses such parallelization technologies as OpenMP and NVIDIA CUDA [5, 6].
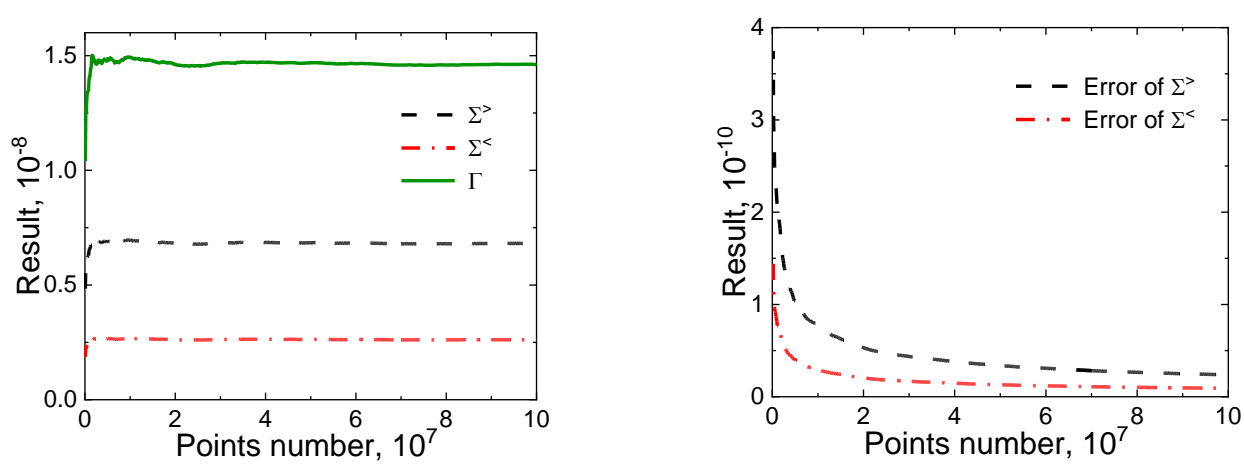

Figure 2. Left panel: $\Gamma, \Sigma^{>}, \Sigma^{<}$as a function of the number of selected points. Right panel: upper error bound as a function of the number of selected points. 
The tests are performed at a fixed temperature $\mathrm{T}=0.15 \mathrm{GeV}, m_{\pi}=0.143 \mathrm{GeV}$ and a fixed amplitude $|\mathrm{A}|=1$ for a different number of selected points. Fig. 2 (left) shows the results of calculations of $\Gamma, \Sigma\rangle, \Sigma$ as a function of the number of selected points. Fig. 2 (right) illustrates the upper error bounds. As seen in Fig. 2 (left), after the limit of $3-4 \cdot 10^{7}$ points, the result becomes insensitive to an increase in the number of calculations, which is caused by a decrease of the upper error bounds.

To speed up calculations, parallelization technologies are used. Table 1 shows the computation time with the generation of $2 * 10^{7}$ points for a different number of threads. The calculations are also performed on the HybriLIT heterogeneous platform of the Meshcheryakov Laboratory of Information Technologies, JINR, Dubna [7]. As demonstrated in Table 1, the use of HybriLIT parallelization resources extremely reduces the computation time.

Table 1. Comparison of the computation time using OpenMP, CUDA and hybrid CUDA+OpenMP parallelization. The number of selected points is equal to $2 \cdot 10^{7}$.

\begin{tabular}{|c|c|c|c|c|c|c|c|c|c|c|}
\hline & None & \multicolumn{3}{|c|}{ OpenMP } & \multicolumn{3}{|c|}{ CUDA } & \multicolumn{3}{|c|}{ CUDA+OpenMP } \\
\hline Threads & - & 4 & 32 & 64 & $8 / 64$ & $16 / 128$ & $32 / 256$ & $8 / 64+4$ & $16 / 128+16$ & $32 / 256+32$ \\
\hline Time, sec & 42 & 8.95 & 2.09 & 1.89 & 3.21 & 0.83 & 0.51 & 2.01 & 0.79 & 0.50 \\
\hline
\end{tabular}

\section{Pion damping}

The physical background of this work is the calculation of the pion lifetime in hot nuclear matter. The amplitude of pion scattering is defined within the NJL model with meson-exchange diagrams considered in the pole approximation, which leads to amplitudes similar to the linear sigma model [8] with isospin amplitudes:

$$
\begin{gathered}
A_{0}=5 C_{4 \pi}+g_{\sigma q q}^{2} g_{\sigma \pi \pi}^{2}\left(\frac{3}{M_{\sigma}^{2}-s}+\frac{1}{M_{\sigma}^{2}-t}+\frac{1}{M_{\sigma}^{2}-u}\right), \\
A_{1}=g_{\sigma q q}^{2} g_{\sigma \pi \pi}^{2}\left(\frac{1}{M_{\sigma}^{2}-t}-\frac{1}{M_{\sigma}^{2}-u}\right), \\
A_{2}=2 C_{4 \pi}+g_{\sigma q q}^{2} g_{\sigma \pi \pi}^{2}\left(\frac{1}{M_{\sigma}^{2}-t}+\frac{1}{M_{\sigma}^{2}-u}\right),
\end{gathered}
$$

where all parameters, the meson mass $M_{\sigma}$ and interaction constants $g_{\sigma \pi \pi}, g_{\sigma \mathrm{qq}}$, depend on temperature, and $C_{4 \pi}$ is supposed to be a constant of the 4-pion interaction, $s, t, u$ are the Mandelstam variables. The coupling strength $g_{\sigma \pi \pi}$ is defined as $g_{\sigma \pi \pi}(T, \mu)=2 g_{\sigma q q} g_{\pi q q}^{2} \Gamma_{\sigma \pi \pi}(T, \mu)$, where $\Gamma_{\sigma \pi \pi}(T, \mu)$ is the amplitude of the triangle vertex $\sigma \rightarrow \pi \pi$.

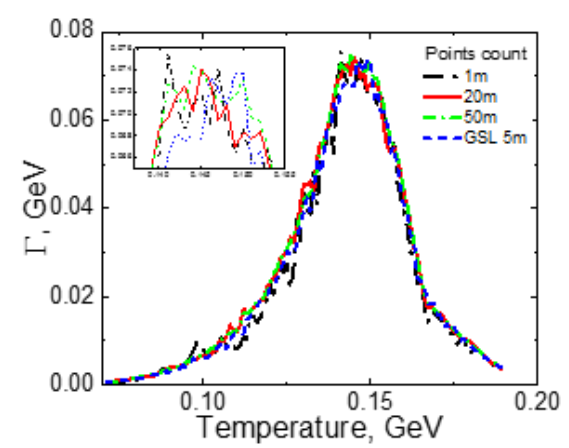

Figure 3. Pion width $(\Gamma)$ as a function of temperature. The results are shown for a different number of selected points and compared with the result of GSL-integration tools (VEGAS) [10].

The temperature dependence for the masses and constants is chosen within the NJL model and can be found, for example, in [9]. In the case of pion-pion collisions, the following processes contribute to the total cross section:

$$
(\pi \pi \longrightarrow \pi \pi)=\sigma\left(\pi^{0} \pi^{0} \longrightarrow \pi^{0} \pi^{0}\right)+\sigma\left(\pi^{0} \pi^{0} \longrightarrow \pi^{+} \pi^{-}\right)+2 \sigma\left(\pi^{0} \pi^{+} \longrightarrow \pi^{0} \pi^{+}\right) .
$$


The process $\pi^{0} \pi^{+} \rightarrow \pi^{0} \pi^{+}$occurs at the same rate as $\pi^{0} \pi^{-} \rightarrow \pi^{0} \pi^{-}$, so we do not calculate them separately; we just put the factor 2. The calculation result is given in Fig. 3. It is clearly seen, that in a hot gas, the pion spectral function significantly broadens (due to an increase in the width) near the critical temperature, which is about $0.17 \mathrm{GeV}$ for this version of the NJL model.

\section{Conclusion}

An algorithm for calculating the pion lifetime as a function of temperature is presented. Since the integrals (2) have a complicated integrand function, it is necessary to select a large number of points, which leads to an increase in the computation time.

To minimize the error, tests with a constant amplitude and a fixed temperature are performed (Fig. 2). The goal of the tests is to identify the minimal number of points for the initial step to plot the pion width as a function of temperature (Fig. 3). However, it cannot be argued that the test results obtained at a fixed temperature are relevant for all temperatures and a non-constant amplitude. Therefore, for each subsequent temperature, an additional calculation is carried out. The calculation with an increasing number of points (with the increase step $\mathrm{M}$ ) is performed until the error obtained in the current iteration becomes worse than in the previous one. Nevertheless, it is difficult to obtain a smooth behavior of the function $\Gamma(T)$ near critical temperatures (Fig. 3). Fig. 3 shows a comparison of our results with GSL-integration tools (VEGAS) [10].

To optimize the computation time, integral optimization and parallelization technologies (such as OpenMP, CUDA and their combination) are applied to the code. The use of the resources of the HybriLIT cluster reduces the computation time and enhances code efficiency.

\section{Acknowledgement}

The work was supported by the RFBR, grant No. 18-02-40137.

\section{References}

[1] L .P. Kadanoff and G. Baym, Quantum Statistical Mechanics //W. A. Benjamin, Icn., New York, 1962.

[2] D. Blaschke, M. K. Volkov, V. L. Yudichev, Pion damping width from SU (2) $\times$ SU (2) NJL model. // Phys.Atom.Nucl. vol. 66, p. 2233-2237, 2013 (nucl-th: 0303034).

[3] I. Sobol. Numerical Monte Carlo methods // Nauka Publishing. 1973, Moscow. (in Russian)

[4] H. Bauke. Tina's Random Number Generator Library Version 4.24, March 27, 2021. Available at: https://www.numbercrunch.de/trng/trng.pdf.

[5] A.S. Antonov. MPI and OpenMP parallel programming technologies: A textbook for universities. Lomonosov Moscow State University; Supercomputer Consortium of Russian Universities. - Moscow: Moscow University Press, 2012. - 344 p. (in Russian)

[6] CUDA C++ Programming Guide - Design Guide, May 2021. Avaliable at: https://docs.nvidia.com/cuda/cuda-c-programming-guide/index.html

[7] Heterogeneous Computing Cluster HybriLIT*. *Available at: //hybrilit.jinr.ru

[8] S. R. Colanch, P. Maris, QCD based quark description of pi-pi scattering up to the sigma and rho region // Phys. Rev. D 66, 116010, 2002. (hep-ph: 0210151).

[9] Yu. L. Kalinovsky and A. V. Friesen, Properties of Mesons and Critical Points in the NambuJona-Lasinio Model with Different Regularizations// Phys. Part. Nucl. Lett., vol. 12, p. 737, 2015.

[10] GNU Scientific Library. Available at: //gnu.org/software/gsl/doc/html/montecarlo.html 